\title{
The analysis of quantitative variation in natural populations with isofemale strains
}

\author{
A.A. HOFFMANN and P.A. PARSONS \\ Department of Genetics and Human Variation, La Trobe University, \\ Bundoora 3083, Victoria, Australia
}

\begin{abstract}
Summary
Isofemale strains are having an increasing role in the analysis of variability of ecological and behavioural traits in natural populations. This paper therefore considers the association between heritability and phenotypic variation within and between isofemale strains. Heritability from an isofemale strain analysis approximates narrow heritability over a wide range of dominance values, particularly when genes contributing to variation in a trait are at intermediate frequencies. Meaningful heritability estimates require that isofemale strains are maintained at a population size greater than 50 and tested within 5 generations after establishment. Values of heritabilities for morphological traits in Drosophila melanogaster were similar to those estimated from a conventional sib analysis. Published data on isofemale strains can therefore be put into a theoretical framework. The contribution of isofemale strain analyses to the debate about the number of loci affecting variation in quantitative traits is briefly discussed.
\end{abstract} cal trait.

Keys words : isofemale strain, heritability, Drosophila melanogaster, gene number, morphologi-

\section{Résumé}

Analyse de la variabilité quantitative dans des populations naturelles par des lignées isofemelles

Les lignées isofemelles jouent un rôle croissant dans l'analyse de la variabilité des caractères écologiques et comportementaux dans les populations naturelles. Cet article a trait aux relations existant entre l'héritabilité et la variation phénotypique intra et entre lignées isofemelles. L'héritabilité obtenue par analyse de lignées isofemelles constitue une approximation de l'héritabilité au sens étroit pour une large gamme de valeurs de dominance, en particulier lorsque les gènes contribuant à la variabilité du caractère ont des fréquences intermédiaires. Une estimation pertinente de l'héritabilité nécessite que les lignées isofemelles soient maintenues à un effectif de population supérieur à 50 et analysées au cours des cinq générations suivant leur fondation. Les valeurs d'héritabilité qui ont été obtenues pour des caractères morphologiques chez Drosophila melanogaster étaient similaires à celles estimées par l'analyse conventionnelle de germains. Les données publiées sur des lignées isofemelles peuvent donc être introduites dans un cadre théorique. La contribution des analyses de lignées isofemelles au débat sur le nombre de locus affectant la variabilité de caractères quantitatifs est brièvement discutée.

Mots clés : lignée isofemelle, héritabilité, Drosophila melanogaster, nombre de gènes, caractère morphologique. 


\section{Introduction}

Isofemale strains have an important role in the assessment of the nature and range of phenotypic variation in natural populations. While these strains were initially used to study quantitative variation in morphological traits, they are of particular value for traits involved in determining the distribution and abundance of organisms (PARSons, 1983). A major advantage of isofemale strains is that genetic information is obtainable in species where details of the genome are not well known. This enables comparative assessments of the evolutionary significance of polygenic variation in species where the underlying genes cannot be readily located due to inadequate linkage maps (PARsons, $1980 a$ ).

From an analysis of variance within and between isofemale strains, estimates of the relative proportions of genetic and environmental variances in populations can be obtained. Such analyses have been useful in comparative studies of populations from different habitats (PARSONS, 1980b) and in comparing different traits (Rockwell et al., 1975). However, it is important to relate these estimates to the heritability of the quantitative characters.

The major aim of this paper is to examine how variation among isofemale strains may be used to estimate heritability. It will be shown that a standard procedure of analysing isofemale strain variation within a few generations of laboratory culture can lead to useful information about narrow heritability, providing that the effective population size of each strain is kept fairly large. Experimental data for morphological traits in Drosophila melanogaster will be used to compare heritability estimates from isofemale strains with those obtained with a sib analysis.

A subsidiary aim is to consider the contribution that isofemale strain analysis can make to the debate (Lande, 1983 ; Gotrlieb, 1984 ; Coyne \& Lande, 1985) about the number and effect of loci that control quantitative variation.

\section{Theory}

\section{A. Full sib analysis and variation among isofemale strains}

Perhaps the simplest way to consider the association between genetic variation and variation among isofemale strains is to view the strains as a series of full-sib families. This approach was used by DAVID (1979) when characterizing morphological variation among progeny from wild-collected females. Progeny are cultured under similar laboratory conditions, so variation among lines (families) is mostly genetic. In an analysis of variance (ANOVA), the between-line component of variance contains a quarter of the additive genetic variance and a quarter of the dominance variance, and the within strain component contains half the additive genetic variance and three quarters of the dominance variance. Hence the intraclass correlation represents half the heritability if only additive effects are present, and somewhat less than half the broad heritability if dominance is present (FALCONER, 1981, p. 156).

Unfortunately, most studies of quantitative genetic variation employing isofemale strains are based on lines kept in the laboratory for several generations (usually $<10$ ). 
Scoring strains in later generations has a number of advantages. Many traits cannot be measured in the first generation because large numbers of individuals may be required from each isofemale strain. Tests on later generations enable strains from populations collected at different times to be compared. Repeat testing of the same strains helps to ensure that differences among them are stable and not due to variation in laboratory culture conditions. Isofemale strains can also be used to obtain estimates of common environment effects which cannot be ascertained in a full-sib analysis.

\section{B. Variation among strains expanded to an infinite size}

To examine the distribution of genetic variance within and between isofemale strains, we first consider the case where strains are expanded to an infinite size after being established from the progeny of a single mating pair. This situation may be approximated by studies with insects such as Drosophila melanogaster which have a high rate of reproduction, enabling the rapid expansion of families into large populations.

TABLE 1

Frequencies of phenotypically different isofemale strains

(for a one locus, two allele trait at Hardy-Weinberg equilibrium in the base population)

\begin{tabular}{l|c|c|c}
\hline \hline $\begin{array}{c}\text { Genotypes } \\
\text { of founder parents }\end{array}$ & Frequency & $\begin{array}{c}\text { Mean score } \\
\text { (no dominance) }\end{array}$ & $\begin{array}{c}\text { Mean score } \\
\text { (dominance) }\end{array}$ \\
\hline 1. $B B \times B B \ldots \ldots \ldots$ & $\mathrm{p}^{4}$ & $\mathrm{a}$ & $\mathrm{a}$ \\
2. $B B \times B b \ldots \ldots$ & $4 \mathrm{p}^{3} \mathrm{q}$ & $\frac{1}{2} \mathrm{a}$ & $\frac{1}{2} \mathrm{a}+\frac{6}{16} \mathrm{~d}$ \\
3. $B b \times B b, \quad B B \times b b$ & $6 \mathrm{p}^{2} \mathrm{q}^{2}$ & 0 & $\frac{1}{2} \mathrm{~d}$ \\
4. $B b \times b b \quad \ldots \ldots$ & $4 \mathrm{pq}^{3}$ & $-\frac{1}{2} \mathrm{a}$ & $-\frac{1}{2} \mathrm{a}+\frac{6}{16} \mathrm{~d}$ \\
$5 . b b \times b b \ldots \ldots$ & $\mathrm{q}^{4}$ & $-\mathrm{a}$ & $-\mathrm{a}$ \\
\hline \hline
\end{tabular}

Consider a single locus with two alleles $(B, b)$, at Hardy-Weinberg equilibrium in the base population. Table 1 shows phenotypic frequencies and mean scores of progeny for each kind of isofemale strain. Matings $B B \times b b$ and $B b \times B b$ have been combined because these result in isofemale strains with the same gene frequency. This table is similar to table 9.2 of FALCONER (1981, p. 138) except that mean scores (M) have been computed after strains have expanded and genotypes are in Hardy-Weinberg within each isofemale strain. The expression used is $\mathbf{M}=a(p-q)+2 d p q$ (FAlconer, 1981, p. 102). Total genetic variance $\left(V_{G}\right)$ within each line has been computed by $\mathrm{V}_{\mathrm{G}}=2 \mathrm{pq}[\mathrm{a}+\mathrm{d}(\mathrm{q}-\mathrm{p})]^{2}+(2 \mathrm{pqd})^{2}$ (FALCONER, 1981, p. 117). In the case of no dominance the mean genetic variance within strains is given by :

$$
V_{w}=p^{4}(0)+4 p^{3} q\left(\frac{3}{8} a^{2}\right)+6 p^{2} q^{2}\left(\frac{1}{2} a^{2}\right)+4 p q^{3}\left(\frac{3}{8} a^{2}\right)+q^{4}(0)
$$




$$
\begin{aligned}
& =\frac{3}{2} \mathrm{pqa}^{2}\left(\mathrm{p}^{2}+2 \mathrm{pq}+\mathrm{q}^{2}\right) \\
& =\frac{3}{2} \mathrm{pqa}^{2}
\end{aligned}
$$

Since the additive genetic variation is $V_{A}=2$ pqa $^{2}$ (FALCONER, 1981, p. 116), then $\mathrm{V}_{\mathrm{w}}=3 / 4 \mathrm{~V}_{\mathrm{A}}$ when summed over all loci. To obtain the variance between strains $\left(\mathrm{V}_{\mathrm{B}}\right)$ the mean of all lines is first obtained as :

$$
\begin{aligned}
M & =p^{4} a-q^{4} a-2 p q^{3} a+2 p^{3} q a \\
& =a(p-q)
\end{aligned}
$$

with simplification. The between strain variance then becomes :

$$
\begin{aligned}
V_{B}= & p^{4}(a-a p+a q)^{2}+q^{4}(-a-a p+a q)^{2}+4 p q^{3}\left(-\frac{1}{2} a-a p+a q\right)^{2} \\
& +6 p^{2} q^{2}(-a p+a q)^{2}+4 p^{3} q\left(\frac{1}{2} a-a p+a q\right) \\
= & a^{2} p q \\
= & \frac{1}{2} V_{A}
\end{aligned}
$$

when summed over all loci. These variance components are obtained in this way to illustrate their relationship to gene frequencies. They could have been obtained more directly from the partitioning of variance within and between strains using the inbreeding coefficient F (FAlCONER, 1981, p. 241) i.e.,

$$
\begin{aligned}
& \mathrm{V}_{\mathrm{B}}=(2 \mathrm{~F}) \mathrm{V}_{\mathrm{A}} \\
& \text { and } \\
& \mathrm{V}_{\mathrm{w}}=(1-\mathrm{F}) \mathrm{V}_{\mathrm{A}}
\end{aligned}
$$

Isofemale strains expanded to an infinite population size have an inbreeding coefficient of $1 / 4$ due to sib-mating during the establishment of a strain, which leads to the $V_{w}$ and $V_{B}$ estimates in (1) and (2).

The isofemale heritability of PARSONS (1983) which corresponds to the intraclass correlation for isofemale strains is defined as :

isofemale heritability $=V_{B} /\left(V_{B}+V_{w}\right)$

$$
=\frac{1}{2} \mathrm{~V}_{\mathrm{A}} /\left(\frac{5}{4} \mathrm{~V}_{\mathrm{A}}+\mathrm{V}_{\mathrm{E}}\right)
$$

where $V_{E}$ is the environmental variance. Using the definition heritability $=h^{2}=V_{A}$ ' $\left(V_{A}+V_{E}\right)$, the relationship between isofemale heritability and actual heritability is defined by :

isofemale heritability $=\frac{1}{2} V_{A} /\left(\frac{5}{4} V_{A}+\frac{V_{A}}{h^{2}}-V_{A}\right)$

$$
=\frac{1}{2} /\left(\frac{1}{4}+\frac{1}{h^{2}}\right) \text {. }
$$


The effect of dominance (d) on the means of isofemale strains is outlined in table 1 . The variance within each strain is defined by $2 p q[a+d(q-p)]^{2}+(2 p q d)^{2}$ (FALCONER, 1981, p. 117), so the total variance within strains (substituting the relevant gene frequencies) becomes

$$
V_{w}=\frac{4}{3} p q\left[2 a^{2}+4 d^{2} p q+\frac{5}{4} p^{2} d^{2}+\frac{5}{4} d^{2} q^{2}-2 a d p^{2}+2 a d q^{2}\right]
$$

The mean of all the strains is :

$$
\begin{aligned}
m & =p^{4}(a)-q^{4}(a)+\frac{3}{2} p q^{3} d+3 p^{2} q^{2} d+2 p^{3} q a+\frac{3}{2} p q^{3} d-2 p q^{3} a \\
& =a(p-q)+\frac{3}{2} p q d
\end{aligned}
$$

so the variance between them is defined by :

$$
\begin{aligned}
V_{B}= & p^{4}(a-m)^{2}+4 p^{3} q\left(\frac{1}{2} a+\frac{6}{16} d-m\right)^{2}+6 p^{2} q^{2}\left(\frac{1}{2} d-m\right)^{2} \\
& +4 p q^{3}\left(-\frac{1}{2} a+\frac{6}{16} d-m\right)^{2}+q^{4}(-a-m)^{2} .
\end{aligned}
$$

Equations (5) and (6) were used to examine the effect of dominance on the heritability estimates calculated from isofemale strains for a range of gene frequencies and dominance values. The broad heritability was set at 0.5 , and the value of « $\mathrm{a}$ " was set to 1.0 , so $\mathrm{d}=1.0$ represents complete dominance for high values of the trait. Overall, heritability estimates from isofemale strains tend to follow narrow heritabilities at intermediate $(p=0.3-0.7)$ or extreme $(p, q<0.05)$ gene frequencies, but deviate from these at other frequencies. Some examples are graphed in figure 1.

\section{Isofemale strains maintained at a finite population size}

Inbreeding will increase divergence among isofemale strains if they are maintained at small population sizes or kept in the laboratory for a number of generations before testing. These effects can be characterized by the relationship between the population size (N) and the inbreeding coefficient (FALCONER, 1981, p. 59), where :

$\mathbf{F}_{\mathbf{t}}=\frac{1}{2 \mathrm{~N}}+\left(1-\frac{1}{2 \mathrm{~N}}\right) \mathbf{F}_{\mathbf{t}-1}$

The graphs in figure 2 were produced by calculating the variance components between and within isofemale strains from the inbreeding coefficient. This assumes that the trait shows only additive genetic variance. Overall, there is little change in the heritability from isofemale strains over 5 generations when strains are kept at an effective population size of 50 or more. However, when they are kept at smaller sizes or for more generations, then the heritability from isofemale strains will overestimate the actual heritability. 

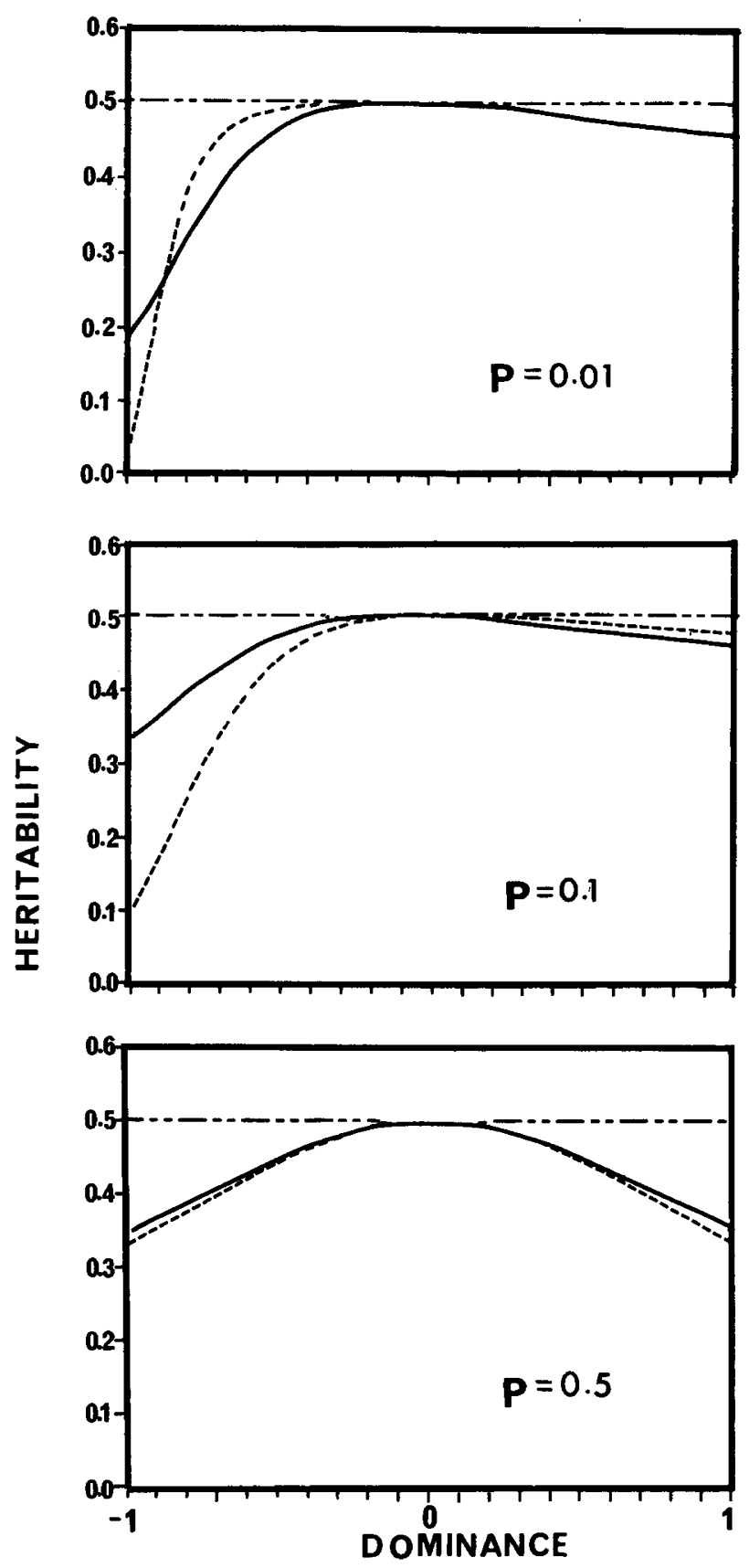

Fig. 1

Effect of dominance on heritability estimates from variation among isofemale strains.

The broad heritability is set at 0.5 , and $p$ defines the frequency of the gene(s) which increases the trait. Lines represent broad heritability (- - -), narrow heritability (- .-) and heritability from isofemale strains (---). 


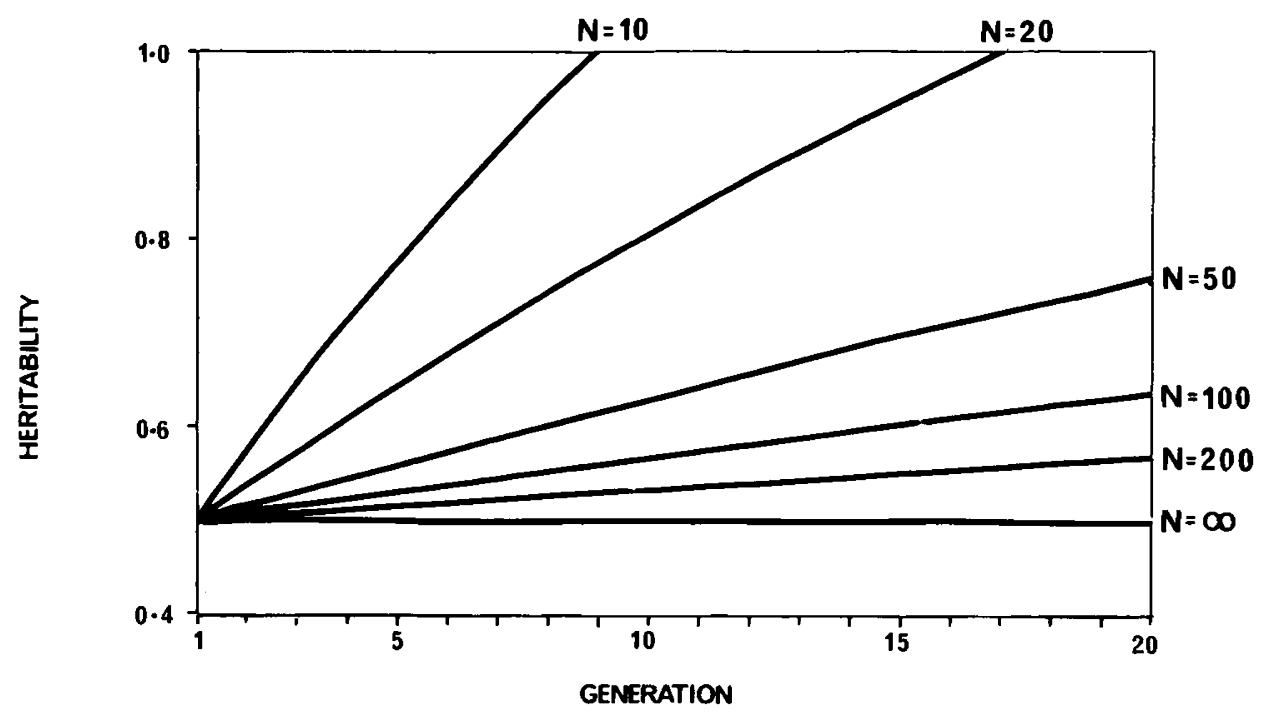

FIG. 2

Heritability estimates from isofemale lines kept at different population sizes $(N)$.

\section{Heritability for traits measured on groups}

Isofemale strains have been used to characterize variation in a number of traits measured on groups of individuals. This includes ecological phenotypes such as desiccation resistance, ethanol tolerance and utilization, and temperature resistance, as well as several behavioural traits (reviews PARSONS, 1980a; 1983). Some of these traits are scored as the mean value for a group, such as percent mortality for desiccation resistance and mean lethal time $50 \%$ for ethanol tolerance.

When an ANOVA is based on groups of individuals, the variance component within groups is reduced, which increases the intraclass correlation. It can be shown that the Mean Square (MS) between groups and MS within groups are decreased by a factor of $1 / x$ where $x$ represents the group size. This means that the "isofemale heritability » for measurements on groups is defined as :

isofemale heritability $=S_{A}^{2} /\left(S_{A}^{2}+x^{2}\right)$

where $S_{A}^{2}$ is the variance component between strains and $S^{2}$ the variance component within strains. Hence measurements which represent the means of groups of individuals can lead to erroneous heritability estimates. This explains some of the high " isofemale heritabilities " presented in PARSONS (1983, p. 53), which are intraclass correlations not adjusted for group size. For example, the appropriate adjustment for ethanol tolerance gives a heritability estimate of 0.84 for the Townsville population and estimates of 0.11 0.17 for the other populations.

These values may still represent overestimates because individuals in the same groups may experience a more similar environment than individuals in other groups. 
This is likely in the case of ethanol tolerance, where groups are exposed to ethanol fumes in sealed containers and different conditions may exist in the containers. However, this problem may not apply to other traits such as desiccation resistance and radiation resistance, where all individuals are simultaneously exposed to the environmental stress (e.g., PARsons, $1970 ; 1975$ ). It should be emphasized that the estimates in Parsons (1983) were used for comparative purposes only, and the evolutionary conclusions presented are valid.

\section{Experimental data}

\section{A. Methods}

We compared heritabilities from an isofemale strain analysis with estimates from a conventional sib analysis using four morphological traits in Drosophila melanogaster: left and right sternopleural bristle number, and wing length and wing width. A laboratory population was established with 60 flies collected from outside a winery at Chateau Tahbilk, Victoria. This population was maintained on a sucrose laboratory medium for three generations at a census size of more than 300 adults. Twenty-one isofemale strains were then established by collecting virgin males and females, and mating them pairwise in vials. By transferring the flies to successive vials, more than 90 progeny were obtained from most of the pairs in the next generation. These flies were maintained in bottles for a further two generations before the traits were scored. Census size was kept at 200-250 individuals. Virgin females and males were collected from the base population for the sib analysis at the time that the isofemale strains were set up. Twenty-one males were each mated to four females and the traits were measured on male progeny from these adults.

Flies to be measured were reared at $20^{\circ} \mathrm{C}$ under controlled low-density conditions. This was achieved by placing twenty eggs in a vial with $10 \mathrm{ml}$ of the laboratory medium. To test for a common environment effect, two vials were set up for each full sib family, and four males were measured from each vial. For the isofemale analysis, four vials were set up per strain, and five males were measured per vial.

Flies were aged for 2-4 days after emergence and placed individually into stoppered tubes. The tubes were stored in a freezer until measurements were made. Sternopleural bristles were counted first, and flies were then mounted on microscope slides for wing removal. Wings were prepared for measurement by laying them on two-sided sticky tape and covering them with a coverslip. Wing images from a video camera were measured on a horizontal surface. Wing length was taken from the intersection of the anterior cross vein and longitudinal vein 3 (L3) to the intersection of L3 with the distal wing margin. Wing width was taken as the distance from the intersection of L5 and the wing margin to the intersection of $\mathrm{L} 2$ and the wing margin.

\section{B. Results}

The traits did not show a significant departure from normality, so the data were analysed without transformation. Variance components were estimated with the SAS 
TABLE 2

Analysis of sib and isofemale data for sternopleural bristle counts, wing width and wing length

\begin{tabular}{|c|c|c|c|c|c|}
\hline & $\begin{array}{l}\text { Left bristle } \\
\text { count }\end{array}$ & $\begin{array}{l}\text { Right bristle } \\
\text { count }\end{array}$ & $\begin{array}{c}\text { Total bristle } \\
\text { count }\end{array}$ & Wing length & Wing width \\
\hline \multicolumn{6}{|l|}{ Sib analysis } \\
\hline \multicolumn{6}{|l|}{ Variance components $(\%)$} \\
\hline- sire $(\mathrm{df}=20)$ & $15^{* *}$ & $9^{* *}$ & $16^{* *}$ & $5^{*}$ & 2 \\
\hline- dam $(\mathrm{df}=55)$ & $14^{* *}$ & $12^{* *}$ & $15^{* *}$ & 1 & 2 \\
\hline- vial $(\mathrm{df}=69)$ & 0 & 0 & 0 & 1 & 0 \\
\hline - error $(\mathrm{df}=466)$ & 71 & 79 & 69 & 93 & 96 \\
\hline \multicolumn{6}{|l|}{ Heritabilities (SE) } \\
\hline - narrow . . & $.46(.15)$ & $.31(.13)$ & $.65(.18)$ & $.09(.07)$ & $.09(.07)$ \\
\hline - broad $\ldots \ldots \ldots$ & $.44(.11)$ & $.37(.10)$ & $.61(.13)$ & $.10(.02)$ & $.08(.05)$ \\
\hline \multicolumn{6}{|l|}{ Isofemale strain analysis } \\
\hline \multicolumn{6}{|l|}{ Variance components (\%) } \\
\hline - strain $(\mathrm{df}=20) \ldots \ldots$ & $19^{* *}$ & $18^{* *}$ & $26^{* *}$ & 4 & $5^{*}$ \\
\hline- vial $(\mathrm{df}=57)$ & 6 & $9^{*}$ & $9^{*}$ & 5 & 0 \\
\hline - error $(\mathrm{df}=245) \quad \ldots$ & 75 & 73 & 65 & 91 & 95 \\
\hline \multicolumn{6}{|l|}{ Heritabilities (SE) } \\
\hline - intraclass correlation & $.19(.06)$ & $.18(.06)$ & $.26(.07)$ & $.04(.03)$ & $.05(.03)$ \\
\hline 一 heritability .... & $.43(.14)$ & $.40(.14)$ & $.60(.16)$ & $.08(.07)$ & $.10(.05)$ \\
\hline
\end{tabular}

Significance levels for $F$ tests : ${ }^{* *} P<0.01 ;{ }^{*} P<0,05$.

VARCOMP procedure. This computes Type I sums of squares for the independent variables (SA $\dot{S}, 1982)$. The overall model was

$$
Y_{i j k}=u+S_{i}+d_{j(i)}+V_{k(i j)}+e_{(i j k)}
$$

where $u$ is the mean, $S_{i}$ the sire effect, $d_{j(i)}$ the nested dam effect, $V_{k(i j)}$ the vial effect nested in dam and sire and $e_{(i j k)}$ the error term. Variance components for the effects are expressed as percentages and are presented in table 2, together with significance levels from $F$ tests.

Heritabilities and standard errors for the sib analysis were estimated according to the methods in FALCONER (1981). Intraclass correlations ( $t$ ) were obtained for the isofemale strain analysis, and standard errors for the correlations were calculated using the method of Osborne \& Paterson (1952). Heritabilities for the isofemale data were calculated using the following equation derived from (3) :

heritability $\left(h^{2}\right)=2 /(1 / t-1 / 2)$.

The standard errors for these heritability estimates were obtained from the standard errors of the intraclass correlation using the approximate formula :

$\operatorname{SE}\left(h^{2}\right)=\frac{2}{(1-t / 2)^{2}} \operatorname{SE}(t)$ 
Heritability estimates from the sib analysis indicate that none of the traits show dominance and common environment (vial) effects are not present (table 2). Narrow heritabilities are intermediate for sternopleural bristle counts and low for the wing measurements. The estimate for total bristle count is consistent with other published values (e.g. GAllego \& Lopez-FANJUl, 1983). Analysis of the isofemale data shows small common environment effects for some traits. Heritability estimates from the isofemale data are similar to those from the sib analysis for all the traits. Thus the isofemale strain analysis provides heritabilities which are consistent with those from the more conventional approach.

\section{Isofemale strain variation and the number of loci affecting quantitative variation}

Data from several isofemale strain studies have been interpreted as indicating that a large percentage of the quantitative variation in morphological and environmental stress traits may be controlled by a few predominantly additive genes of large effect (e.g., Parsons, 1980a ; Thompson \& Hellack, 1982 ; Thompson \& Mascie-Taylor, 1985 ; THOMPSON et al., 1986).

In selection experiments on scutellar bristle number in $D$. melanogaster, directional selection produced a rapid response in isofemale strains which had high scores for the trait, but no response in strains which had a 4-bristle score (e.g., HosGood et al., 1968). Considering the one-locus situation described in table 1 , the genotypic combinations in rows 1 and $5(B B \times B B, b b \times b b)$ would not respond to selection. The other three genotypic combinations should respond rapidly, because the rate of response is proportional to the product of the allele frequencies and therefore rapid at the intermediate gene frequencies in these lines. If additional loci are considered with the favoured allele at frequency $p$, then the proportion of lines which do not respond to selection is given by $\left(q^{4}\right)^{n}$ where $n$ is the number of loci. This means that almost all the isofemale strains would respond if $p$ and $n$ were relatively large. For example, if $p=0.2$ and $n=10$, then only $0.0001 \%$ of the lines would not respond to selection. Even if three loci were postulated, only $7 \%$ of the strains would not respond. This finding implies that a few loci may explain response to directional selection if allele frequencies are assumed to be intermediate.

An alternative explanation is that a large number ( $n$ ) of loci contribute to variation in the base population, but favoured alleles are at low frequencies. For example, if $\mathrm{p}=0.01$ and $\mathrm{n}=50$, then $13 \%$ of the strains would not respond to selection. However isofemale strains with favoured alleles would still respond rapidly because the frequency of the favoured allele will always be at least 0.25 in an isofemale strain (table 1). Hence the rapid response of some isofemale strains to directional selection and absence of any response in other strains is consistent with different interpretations which can only be resolved with additional experiments. For example, "identity tests » (Milkman, 1965) could be used to examine whether the same alleles had responded in different populations.

Another finding is that polygene location studies on isofemale strains which have extreme scores for a trait indicate that variation between extreme strains can usually be attributed to one or a few chromosomal segments (e.g., DeERY \& Parsons, 1972). This 
would represent convincing evidence for a few loci if differences among isofemale strains could be repeatedly attributed to the same chromosomal segments. Unfortunately published studies have normally focussed on only two extreme strains. If favoured alleles are rare and many loci are involved, then extreme strains could differ at only a few loci after selection. The same difficulty emerges in interpreting many published experiments on directional and disruptive selection in D. melanogaster which have used small base populations (e.g., Mather \& HARrison, 1949 ; Frazer et al., 1965 ; THODAY \& BoAm, 1959) and are therefore unlikely to have sampled much of the genetic variation for rare alleles. A study of the location of polygenic activity in an array of isofemale strains would therefore be useful.

The demonstration of polygenic segregation within an isofemale strain has been taken as evidence that a few loci are involved in the base population (THOMPSon \& Hellack, 1982). Polygenic segregation within isofemale strains would not be detected if there are numerous loci with relatively common alleles affecting the trait. However, simple segregation patterns will exist if these alleles are rare, because most strains will only segregate for one or two loci. A related finding is that isofemale strains may fall into a limited number of polygenic segregation patterns (THOMPSON \& MASCIE-TAYLOR, 1985 ; THомpson et al., 1986). This observation can also be explained if numerous loci with rare favoured alleles are assumed. Consider $n$ loci with the frequency of the rare allele increasing a trait defined by $\mathrm{p}$. Three types of isofemale strains may be relatively common. The first type has no rare alleles, and its frequency is $\left(q^{4}\right)^{n}$ as above. The second type has segregation at only one locus, and has a frequency of (n) $\left(4 \mathrm{pq}^{3}\right)$ $\left(q^{4}\right)^{n-1}$. The third type has segregation at two loci, and has a frequency of $\left(4 \mathrm{pq}^{3}\right)^{2}$ $\left(q^{4}\right)^{n-2}[(n-2)+(n-3)+\ldots(n-n)]$. These three types of strains will show different segregation patterns, and may account for most of the isofemale strains in a sample. For example, if $n=20$ and $p=0.01$, then they will account for $45 \%, 37 \%$ and $12 \%$ of the strains respectively. Hence a limited number of segregation patterns does not necessarily imply that only a few loci control genetic variation in a trait.

In summary, the patterns of variation within and between isofemale strains suggest that loci of large effect contribute to most phenotypic variation in morphological traits, but it is not clear whether a few polymorphic loci or numerous loci with rare alleles are involved. These alternatives can only be distinguished with additional experiments on the identity of alleles segregating or selected in different isofemale strains.

\section{Concluding remarks}

Isofemale strains provide a relatively simple approach to the assessment of polygenic variation in natural populations. They can provide an initial heritability estimate if strains are tested soon after they are set up and maintained at a fairly large population size. The extensive use of isofemale strains in examining genetic variation for ecological and behavioural traits should provide the basis for a more detailed genetic analysis of these traits.

Received February 27, 1987.

Accepted June 15, 1987. 


\section{Acknowledgements}

We thank Dr. R.G. StaudTe for assistance with statistical matters.

\section{References}

Coyne J.A., Lande R., 1985. The genetic basis of species differences in plants. Amer. Nat., 126, 141-145.

DAvid J.R., 1979. Utilization of morphological traits for the analysis of genetic variability in wild populations. Aquilo Ser. Zool., 2, 49-61.

Deery B.J., Parsons P.A., 1972. Ether resistance in Drosophila melanogaster. Theor. Appl. Genet., 42, 208-214.

FALCONER D.S., 1981. Introduction to quantitative genetics, 2nd ed., 340 p., Longman, London.

Frazer A.S., Scowcroft W., Nassar R., Angeles H., Bravo G., 1965. Variation of scutellar bristles in Drosophila. 4. Effects of selection. Aust. J. Biol. Sci., 18, 619-641.

Gallego A., Lopez-Fanjul C., 1983. The number of loci affecting a quantitative trait in Drosophila melanogaster revealed by artificial selection. Genet. Res., Camb., 42, 137-149.

Gotrlieb L.D., 1984. Genetics and morphological evolution in plants. Amer. Nat., 123, 681-709.

Hosgood S.M.W., MacBean I.T., Parsons P.A., 1968. Genetic heterogeneity and accelerated responses to directional selection in Drosophila. Mol. Gen. Genet., 101, 217-226.

LANDE R., 1983. The response to selection on major and minor mutations affecting a metrical trait. Heredity, 50, 47-65.

Mather K., Harrison B.J., 1949. The manifold effect of selection. Heredity, 3, 1-52, 131-162.

MilKman R.D., 1965. The genetic basis of natural variation. 7. The individuality of polygenic combinations in Drosophila. Genetics, 52, 789-799.

Osborne R., Paterson W.S.B., 1952. On the sampling variance of heritability estimates derived from variance analysis. Proc. Roy. Soc. Edinburgh, 64, 456-461.

Parsons P.A., 1970. Genetic heterogeneity in natural populations of Drosophila melanogaster for ability to withstand desiccation. Theor. Appl. Genet., 40, 261-266.

Parsons P.A., 1975. Differences between Drosophila melanogaster and its sibling species $D$. simulans to radioresistance using isofemale strains from natural populations. Int. J. Radiat. Biol., 27, 297-300.

Parsons P.A., 1980a. Isofemale strains and evolutionary strategies in natural populations. Evol. Biol., 13, 175-217.

Parsons P.A., 1980b. Adaptive strategies in natural populations of Drosophila : ethanol tolerance, desiccation resistance and development times in climatically optimal and extreme environments. Theor. Appl. Genet., 57, 257-266.

Parsons P.A., 1983. The evolutionary biology of colonizing species. 262 p., Cambridge University Press, New York.

Rockwell R.F., Cooke F., Harmsen R., 1975. Photobehavioral differentiation in natural populations of Drosophila pseudoobscura and Drosophila persimilis. Behav. Genet., 5, 189-202.

SAS Institute Inc., 1982. User's Guide : Statistics. 956 p., SAS Inst. Inc., Cary, NC.

Thoday J.M., Boam T.B., 1959. Effects of disruptive selection. 2. Polymorphism and divergence without isolation. Heredity, 13, 205-218.

Thompson J.N., Hellack J.J., 1982. Polygenic segregation within an isofemale strain of Drosophila. Can. J. Genet. Cytol., 24, 235-241.

Thompson J.N., Mascie-Taylor C.G.N., 1985. Detection of simple polygenic segregations in a natural population. Proc. Natl. Acad. Sci. USA, 82, 8552-8556.

Thompson J.N., Hellack J.J., Schnell G.D., 1986. Numerical techniques for the analysis of polygenes sampled from natural populations. Génét. Sél. Evol., 18, 279-298. 